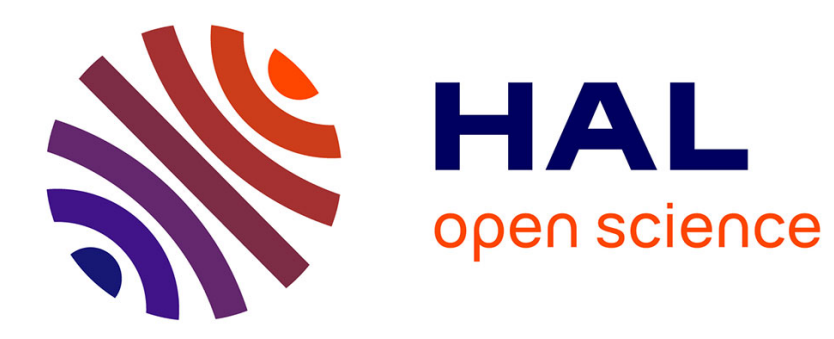

\title{
Weak solution of semi-linear PDE, BSDE and homogenization
}

Antoine Lejay

\section{To cite this version:}

Antoine Lejay. Weak solution of semi-linear PDE, BSDE and homogenization. Monte Carlo and probabilistic methods for partial differential equations (Monte Carlo, 2000), 2000, Monte Carlo, pp.262-272. inria-00101706

\section{HAL Id: inria-00101706 https://hal.inria.fr/inria-00101706}

Submitted on 27 Sep 2006

HAL is a multi-disciplinary open access archive for the deposit and dissemination of scientific research documents, whether they are published or not. The documents may come from teaching and research institutions in France or abroad, or from public or private research centers.
L'archive ouverte pluridisciplinaire HAL, est destinée au dépôt et à la diffusion de documents scientifiques de niveau recherche, publiés ou non, émanant des établissements d'enseignement et de recherche français ou étrangers, des laboratoires publics ou privés. 


\section{Weak solution of semi-linear PDE, BSDE and homogenization}

Antoine Lejay ${ }^{1, \dagger}$ — Projet OMEGA (INRIA / Institut Élie Cartan, Nancy)

Abstract: The sketch of the proof using BSDE of a homogenization result for semi-linear PDE with a divergence-form operator is given here. The method employed here relies on the use of a weak topology.

Keywords: BSDE, homogenization, divergence-form operators, semi-linear PDE

AMS Classification: 35B27 (35K55 35R60 60H15)

Published in Monte Carlo Methods Appl..

Proc. of Monte Carlo and probabilistic methods for partial differential equations, Part II (Monte Carlo, 2000),

7:3-4, 262-272, 2001

Archives, links \& reviews:

o MR Number: 1834783

${ }^{1}$ Current address: Projet OMEGA

Institut Élie Cartan, UMR 7502,

Campus scientifique

BP 239

54506 Vandœuvre-lès-Nancy CEDEx, France

E-mail: Antoine.Lejay@iecn.u-nancy.fr

$\dagger$ This article was written while the author was a preparing his Ph.D. at the SYSDYS team of INRIA Sophia-Antipolis and the Laboratoire d'Analyse, Topology et Probabilités (LATP) of the Université de Provence (Marseille, France). 
A. Lejay / Weak solution of semi-linear PDE, BSDE and homogenization

\section{Introduction}

A Backward Stochastic Differential Equation (BSDE) may be associated to a semi-linear PDE of the type

$$
\left\{\begin{array}{l}
\frac{\partial u(t, x)}{\partial t}+L u(t, x)+h(t, x, u(t, x), \nabla u(t, x))=0,(t, x) \in[s, T] \times \mathcal{O}, \\
u(T, x)=g(x) \text { and } u(t, x)=0 \text { on } \partial \mathcal{O} \text { for any } t \in[s, T)
\end{array}\right.
$$

where $L$ is a second-order differential operator, and $\mathcal{O}$ is an open domain of $\mathbb{R}^{N}$. In some cases, if $\mathrm{X}$ is a stochastic process whose infinitesimal generator is $L$, and if $\mathrm{M}^{\mathrm{X}}$ is its martingale part, there exists a unique couple of processes $\left(\mathrm{Y}_{t}, \mathrm{Z}_{t}\right)_{t \in[s, T]}$ such that

$$
\left\{\begin{array}{l}
\mathrm{Y}_{t}=g\left(\mathbf{X}_{T}^{s, x}\right)+\int_{t}^{T} h\left(r, \mathbf{X}_{r}^{s, x}, \mathbf{Y}_{r}, \mathbf{Z}_{r}\right) \mathrm{d} r-\int_{t}^{T} \mathbf{Z}_{r} \mathrm{dM}_{r}^{\mathrm{X}}, s \leqslant t \leqslant T, \\
\mathbb{E}\left[\sup _{s \leqslant t \leqslant T}\left|\mathrm{Y}_{t}\right|^{2}+\int_{s}^{T}\left\|\mathrm{Z}_{t}\right\|^{2} \mathrm{~d} t\right]<+\infty, \\
\mathbf{Y}_{t} \text { and } \mathbf{Z}_{t} \text { are progressively measurable w.r.t. } \sigma\left(\mathbf{X}_{r}^{s, x}, s \leqslant r \leqslant t\right) .
\end{array}\right.
$$

The processes $\mathrm{Y}$ and $\mathrm{Z}$ are adapted to the filtration generated by $\mathrm{X}^{s, x}$. The final value $Y_{T}$ is known, and we are interested in finding the initial value of $Y$. This value $\mathbf{Y}_{0}$ is $\sigma\left(\mathbf{X}_{s}\right)$-adapted, hence deterministic if $\mathbf{X}_{s}^{s, x}=x$. The relation between (2) and (3) has been first established by É. Pardoux and S. Peng in [PP90], and is

$$
\mathrm{Y}_{t}=u\left(t, \mathrm{X}_{t}^{s, x}\right) \text { and } \mathrm{Z}_{t}=\nabla u\left(t, \mathrm{X}_{t}^{s, x}\right) \text { for } s \leqslant t \leqslant T .
$$

In particular, the relation (3) implies that

$$
u(s, x)=\mathrm{Y}_{s} \text { if } \mathrm{X}_{s}^{s, x}=x .
$$

The solution of the BSDE (4) gives the solution of the semi-linear PDE, and (2) is sometimes called the non-linear Feynman-Kac formula.

In a decade, the interest on BSDEs, with or without direct relation to PDE, has grown very quickly, with some fields of applications as finance and stochastic control. In cite [EK97, PAR99a], the reader may find some introduction to the theory of BSDE.

We give here one of the way to prove that the solutions $u^{\varepsilon}$ to the semilinear PDEs

$$
\left\{\begin{aligned}
& \frac{\partial u^{\varepsilon}(t, x)}{\partial t}+ L^{\varepsilon} u^{\varepsilon}(t, x)+h\left(t, x / \varepsilon, x, u^{\varepsilon}(t, x)\right) \\
&+\nabla u^{\varepsilon}(t, x) \widehat{h}\left(t, x / \varepsilon, x, u^{\varepsilon}(t, x)\right)=0,(t, x) \in[s, T] \times \mathcal{O}, \\
& u^{\varepsilon}(T, x)= g(x), \\
& u^{\varepsilon}(t, x)=0 \text { on } \partial \mathcal{O} \text { for any } t \in[s, T),
\end{aligned}\right.
$$


converge, where $L^{\varepsilon}$ is a divergence-form operator

$$
L^{\varepsilon}=\frac{1}{2} \frac{\partial}{\partial x_{i}}\left(a_{i, j}(x / \varepsilon) \frac{\partial}{\partial x_{j}}\right),
$$

and where the coefficient $a$ and the functions $x \mapsto h(\cdot, x, \cdot, \cdot)$ and $x \mapsto$ $\widehat{h}(\cdot, x, \cdot, \cdot)$ are either periodic or correspond to stationary random field of an ergodic random media. In this case, the limit $\bar{u}$ of the sequence $\left(u^{\varepsilon}\right)_{\varepsilon>0}$ is also the solution of some semi-linear PDE. This function gives the statistical behavior of the media represented by the PDE (5) in a macroscopic scale. Such a result is called an homogenization property, and is inscribed in the homogenization theory, whose goal is to approximate highly-oscillating medias by some simpler one.

The convergence of the solutions of equation (5) may be proved with analytical methods [BLP78], but it may also be proved using the corresponding BSDE. We shall prove the convergence of the processes $X^{\varepsilon}$ whose infinitesimal generator is $L^{\varepsilon}$, and identify the limit. In fact, the convergence follows from some ergodic property. We shall then work on the family of BSDE

$$
\left\{\begin{array}{l}
\mathbf{Y}_{t}^{\varepsilon}=g\left(\mathbf{X}_{T}^{\varepsilon, s, x}\right)+\int_{t}^{T} h\left(r, \mathbf{X}_{r}^{\varepsilon, s, x} / \varepsilon, \mathbf{X}_{r}^{\varepsilon, s, x}, \mathbf{Y}_{r}^{\varepsilon}\right) \mathrm{d} r \\
\quad+\int_{t}^{T} \mathbf{Z}_{r}^{\varepsilon} \widehat{h}\left(r, \mathbf{X}_{r}^{\varepsilon, s, x} / \varepsilon, \mathbf{X}_{r}^{\varepsilon, s, x}, \mathbf{Y}_{r}^{\varepsilon}\right) \mathrm{d} r-\int_{t}^{T} \mathbf{Z}_{r}^{\varepsilon} \mathrm{dM}_{r}^{\mathbf{X}^{\varepsilon}}, s \leqslant t \leqslant T, \\
\mathbb{E}\left[\sup _{s \leqslant t \leqslant T}\left|\mathrm{Y}_{t}^{\varepsilon}\right|^{2}+\int_{s}^{T}\left\|\mathbf{Z}_{t}^{\varepsilon}\right\|^{2} \mathrm{~d} t\right]<+\infty, \\
\mathbf{Y}_{t}^{\varepsilon} \text { and } \mathbf{Z}_{t}^{\varepsilon} \text { are progressively measurable w.r.t. } \sigma\left(\mathbf{X}_{r}^{\varepsilon, s, x}, s \leqslant r \leqslant t\right) .
\end{array}\right.
$$

With (4), the convergence of $Y^{\varepsilon}$ to some solution $\bar{Y}$ of some BSDE implies the convergence of $u^{\varepsilon}$ to solution of the correspond semi-linear PDE. We obtain then the pointwise convergence of the $u^{\varepsilon}$, and not functional convergence as with the analytical method.

There exists two families of proofs concerning homogenization property by BSDE. The first one, which will be used here and which has been initiated by É. Pardoux and A.Y. Veretennikov [PV97], consists in proving the convergence of $\mathrm{Y}^{\varepsilon}$ in a weak topology, namely the $S$-topology [JAK97]. Among the related works, we may cite [PAR99b, GAU99, PO99]. The drawback of this method is that it does not allow to consider a general non-linearity in $\nabla u$, except for the special case of a quadratic non-linearity [GAU99].

The other family relies on some stability property for BSDE proved by Y. Hu and S. Peng [HU97, HP97], and is used in [BHP98, BRI97, BH98, CAS00]... This method allows to deal with a term non-linear in $\nabla u$, but requires more regularity on the coefficients. Both methods may also be used to prove convergence results other than homogenization. 
The novelties of the present work are that the semi-linear PDE contains a first-order differential non-linear term, and that the operator is a divergence-form operator without regularity assumption on its coefficient. In this context, the process $\mathrm{X}^{\varepsilon, s, x}$ shall be studied using Dirichlet Form theory [FOT94, MR91], and the solutions $u^{\varepsilon}$ of (5) are weak solutions.

The relation (3) has been developed first in the context of classical solution, when $\mathrm{X}^{s, x}$ is the solution of some stochastic differential equation (SDE). It has been then proved that BSDE also provides viscosity solution to PDE (1) (see e.g., [PAR98]). The key element in both context is the Itô formula applied to $u\left(t, \mathrm{X}_{t}^{s, x}\right)$.

But in the stochastic process generated by a divergence-form operator is in general not the solution of some SDE, and the solution of (1) belongs to the space

$$
\left\{f \in \mathrm{L}^{2}\left(s, T ; \mathrm{H}_{0}^{1}(\mathcal{O})\right) \mid \partial f / \partial t \in \mathrm{L}^{2}\left(s, T ; \mathrm{H}^{-1}(\mathcal{O})\right\}\right.
$$

An account on non-linear parabolic PDE may be found in [LSU68] for example.

The link between BSDE and weak solution of semi-linear PDE has been highlighted first by G. Barles and E. Lesigne [BL97], but in their case, the driving stochastic process is the solution of some SDE. In fact, it may be proved that even if the coefficient $a$ is not regular, then the representation (3) is again valid. The reader is referred to [LEJ00a, LEJ00b], where a regularization argument is used, and to [BPS99], where the Fukushima Stochastic Calculus is extended. The previously cited articles, together with some other works on different types of semi-linear PDE shows that the notion of weak solution provided also a natural framework for BSDEs, although the results did not from a simple application of the Itô calculus.

The remainder of this paper is devoted to the homogenization property for the family of semi-linear PDEs (5). A detailed proof may be found in [LEJ00a, LEJ00b] in periodic and random media.

\section{The homogenization property}

For simplicity reason, we will treat only the case of periodic coefficient. The results given here may be easily proved in the case of the coefficient of the PDE are stationary random fields. We prove an homogenization result for the PDE (5).

We also assume that $\mathcal{O}=\mathbb{R}^{N}$. Else, we have to kill the processes $\mathrm{X}^{\varepsilon}$ when they exit from $\mathcal{O}$. 
A. Lejay / Weak solution of semi-linear PDE, BSDE and homogenization

We assume that

$$
\begin{gathered}
\lambda|\xi|^{2} \leqslant a(x) \xi \cdot \xi \leqslant \Lambda|\xi|^{2} \\
\left|h\left(t, x, x^{\prime}, y\right)-h\left(t, x, x^{\prime}, y^{\prime}\right)\right| \leqslant C\left|y-y^{\prime}\right| \\
h\left(t, x, x^{\prime}, y\right) \text { and } h\left(t, x, x^{\prime}, y\right) \text { are equi-continuous uniformly w.r.t } x \\
\left(t, x, x^{\prime}\right) \mapsto h\left(t, x, x^{\prime}, 0\right) \text { is bounded } \\
\widehat{h} \text { is bounded } \\
x \mapsto h(\cdot, x, \cdot, \cdot), x \mapsto \widehat{h}(\cdot, x, \cdot, \cdot) \text { and } a \text { are periodic }
\end{gathered}
$$

plus some measurability conditions on the coefficients.

We give in the remaining part of this paper the sketch of the proof of the convergence of $u^{\varepsilon}$ to the solution $\bar{u}$ of

$$
\left\{\begin{array}{l}
\frac{\partial \bar{u}(t, x)}{\partial t}+\frac{1}{2} \bar{a}_{i, j} \frac{\partial^{2} \bar{u}(t, x)}{\partial x_{i} \partial x_{j}}+\bar{h}(t, x, \bar{u}(t, x))+\nabla \bar{u} \cdot \overline{\widehat{h}}(t, x, \bar{u}(t, x))=0 \\
\bar{u}(T, x)=g(x) \text { and } \bar{u}(t, x)=0 \text { on }[s, T) \times \partial \mathcal{O}
\end{array}\right.
$$

The coefficient $\bar{a}$ is constants, and all the coefficients of this PDE will be identified later by (17), (18) and (14).

\section{Homogenization of the linear part}

We may assume without loss of generality that $s=0$, and then we drop any further reference to $s$.

We shall first deal we the convergence of the process $\mathbf{X}^{x, \varepsilon}$. In fact,

$$
\mathbf{X}^{x, \varepsilon}=\varepsilon \cdot \mathbf{X}_{\cdot / \varepsilon^{2}}^{x / \varepsilon, 1}
$$

Hence, we have to prove some Central Limit Theorem on the process $X^{x, 1}$. From now, we drop any reference to the starting point, and we set $X=X^{1}$. There are two key points in the proof. The first consists to prove the existence of some functions $u_{i}(x)=x_{i}+v_{i}(x)$ where $v_{i}$ are periodic, bounded continuous functions and such that $L^{\varepsilon} u_{i}^{\varepsilon}=0$ if $u_{i}^{\varepsilon}(x)=\varepsilon u_{i}(x / \varepsilon)$ for $i=1, \ldots, N$. Hence, using the Itô-Fukushima formula [FOT94, Theorem 5.2.2, p. 203]

$$
u_{i}^{\varepsilon}\left(\mathbf{X}_{t}^{\varepsilon}\right)-u_{i}^{\varepsilon}\left(\mathbf{X}_{0}^{\varepsilon}\right)=\widetilde{\mathrm{M}}_{t}^{i, \varepsilon},
$$

where $\widetilde{\mathrm{M}}^{\varepsilon}$ is a local martingale with cross-variations

$$
\left\langle\widetilde{\mathrm{M}}^{i, \varepsilon}, \widetilde{\mathrm{M}}^{j, \varepsilon}\right\rangle \stackrel{\text { law }}{=} \varepsilon^{2} \int_{0}^{t / \varepsilon^{2}}\left\langle a \nabla u_{i}, \nabla u_{j}\right\rangle\left(\mathbf{X}_{s}\right) \mathrm{d} s .
$$


From the boundedness of the function $u_{i}$, we easily see that

$$
u_{i}^{\varepsilon}\left(\mathbf{X}^{\varepsilon}\right)-\mathbf{X}^{\varepsilon} \underset{\varepsilon \rightarrow 0}{\stackrel{\text { proba }}{\longrightarrow}} 0 .
$$

Hence, $\mathrm{X}^{\varepsilon}$ and $u_{i}^{\varepsilon}\left(\mathrm{X}^{\varepsilon}\right)$ will have the same limit in distribution if one of this process converges in distribution. Our Central Limit Theorem has then been transformed in a Central Limit Theorem on local martingales. According to Theorem 7.1.4 in [EK86, p. 339], we have only to prove the convergence in probability of the cross-variations of $\widetilde{\mathrm{M}}^{\varepsilon}$.

Although the process $X$ is not ergodic, its projection on the unit torus $\mathbb{T}^{N}=\mathbb{R}^{N} / \mathbb{Z}^{N}$ is ergodic with respect to the Lebesgue Measure. Since $a$ and $\nabla u_{i}$ are periodic for $i=1, \ldots, N$, the Ergodic Theorem applied to (12) yields

$$
\left\langle\widetilde{\mathrm{M}}^{i, \varepsilon}, \widetilde{\mathrm{M}}^{j, \varepsilon}\right\rangle \underset{\varepsilon \rightarrow 0}{\longrightarrow} t \bar{a}_{i, j}=t \int_{\mathbb{T}^{N}}\left\langle a \nabla u_{i}, \nabla u_{j}\right\rangle(x) \mathrm{d} x .
$$

Hence, if $\bar{\sigma}$ is such that $\bar{\sigma} \cdot \bar{\sigma}^{\mathrm{T}}=\bar{a}$, then $\mathrm{X}^{\varepsilon}$ converges in distribution to the process $\bar{\sigma} \mathrm{B}$, where $\mathrm{B}$ is a standard Brownian Motion.

\section{Convergence of the BSDE}

The core of the proof can now be given. The general idea that come from É. Pardoux \& A.Y. Veretennikov [PV97] consists in using a very weak topology to prove the convergence of $Y^{\varepsilon}$. In fact, proving the convergence of the process $\mathrm{Y}^{\varepsilon}$ in the usual topology of continuous function requires some estimates very hard to establish (see the comments p. 535 in [PAR99a]).

The topology used here is the $S$-topology introduced by A. Jakubowski in [JAK97]. The Meyer-Zheng topology may also have been used, as in [PV97]. The advantage of such topologies is that tightness criterion are easy to prove. However, the tightness of $\left(\mathrm{Y}^{\varepsilon}\right)_{\varepsilon>0}$ is proved, but nothing can be said on the family $\left(Z^{\varepsilon}\right)_{\varepsilon>0}$. In fact, the family of family of martingale parts $\left(\int \mathrm{Z}_{s}^{\varepsilon} \mathrm{dM}_{s}^{\mathrm{X}^{\varepsilon}}\right)_{\varepsilon>0}$ is also tight for the $S$-topology, and no further reference to $Z^{\varepsilon}$ will be done in our proof. This is why this method does not allow to deal with a general non-linear term in $\nabla u^{\varepsilon}$, which corresponds to $Z^{\varepsilon}$.

However, in our problem (see (7)), the non-linear first-order differential term add some term with $\mathrm{Z}^{\varepsilon}$ outside the martingale part of the BSDE. The idea, which presents some novelty with respect to the previous works, consists in using the Girsanov Theorem to transform the BSDE together with the equation of $u^{\varepsilon}\left(\mathrm{X}^{\varepsilon}\right)$ on a system of coupled forward-backward stochastic differential equations (FBSDE). Under the new distribution, the process $Y^{\varepsilon}$ will also be $S$-tight. 
Step 1: The Girsanov transform. We set

$$
\mathrm{L}_{t}^{\varepsilon}=\int_{0}^{t} a^{-1}\left(\mathbf{X}_{s}^{\varepsilon} / \varepsilon\right) \widehat{h}\left(s, \mathrm{X}_{s}^{\varepsilon} / \varepsilon, \mathrm{X}_{s}^{\varepsilon}, \mathrm{Y}_{s}^{\varepsilon}\right) \mathrm{d} s \text { and }\left.\frac{\mathrm{d} \widehat{\mathbb{P}}^{\varepsilon}}{\mathrm{d} \mathbb{P}^{\varepsilon}}\right|_{\mathcal{F}^{\chi^{\varepsilon}}}=\exp \left(\mathrm{L}_{t}^{\varepsilon}-\frac{1}{2}\left\langle\mathrm{~L}^{\varepsilon}\right\rangle_{t}\right)
$$

where $\left(\mathcal{F}_{t}^{\mathbf{X}^{\varepsilon}}\right)_{t \geqslant 0}$ is the filtration generated by $\mathbf{X}^{\varepsilon}$. As $\widehat{h}$ is bounded, with the Cauchy-Schwarz inequality, there exists some constant $C$ independent from $\varepsilon$ such that

$$
\widehat{\mathbb{E}}^{\varepsilon}[\cdot] \leqslant C \mathbb{E}^{\varepsilon}\left[(\cdot)^{2}\right]^{1 / 2} .
$$

In particular, if $K$ is a measurable event such that $\mathbb{P}^{\varepsilon}[K] \underset{\varepsilon \rightarrow 0}{\longrightarrow} 0$, then $\widehat{\mathbb{P}}^{\varepsilon}[K] \underset{\varepsilon \rightarrow 0}{\longrightarrow} 0$.

Step 2: Convergence of $\mathrm{Y}^{\varepsilon}$ and $\mathrm{M}^{\varepsilon}$. We set $\mathrm{M}_{t}^{\varepsilon}=\int_{0}^{t} Z_{s}^{\varepsilon} \mathrm{dM}_{s}^{\mathrm{X}^{\varepsilon}}$. Using standard arguments in BSDE theory,

$$
\sup _{\varepsilon>0} \widehat{\mathbb{E}}\left[\sup _{0 \leqslant t \leqslant T}\left|\mathbf{Y}_{t}^{\varepsilon}\right|^{2}+\sup _{0 \leqslant t \leqslant T}\left|\mathbf{M}_{t}^{\varepsilon}\right|^{2}\right]<+\infty
$$

It follows that $\left(\mathcal{L}\left(\mathrm{X}^{\varepsilon}, \mathrm{Y}^{\varepsilon}, \mathrm{M}^{\varepsilon} \mid \widehat{\mathbb{P}}^{\varepsilon}\right)\right)_{\varepsilon>0}$ is tight for the $U \times S \times S$-topology (the $U$-topology is the uniform topology), and we may extract a convergent subsequence - identified with the whole sequence - to some element $\mathcal{L}(\overline{\mathrm{X}}, \overline{\mathrm{Y}}, \overline{\mathrm{M}} \mid \widehat{\mathbb{P}})$. The processes $\overline{\mathrm{Y}}$ and $\overline{\mathrm{M}}$ are a priori only càdlàg functions.

Step 3: Passage to the limit of $\left(\mathcal{L}\left(X^{\varepsilon} \mid \widehat{\mathbb{P}}^{\varepsilon}\right)\right)_{\varepsilon>0}$. Under the new distribution $\widehat{\mathbb{P}}^{\varepsilon},(11)$ becomes

$$
u_{i}^{\varepsilon}\left(\mathbf{X}_{t}^{\varepsilon}\right)-u_{i}^{\varepsilon}\left(\mathbf{X}_{0}^{\varepsilon}\right)=\widehat{\mathrm{M}}_{t}^{i, \varepsilon}+\int_{0}^{t} \nabla u_{i}\left(\mathbf{X}^{\varepsilon} / \varepsilon\right) \cdot \widehat{h}\left(s, \mathbf{X}_{s}^{\varepsilon} / \varepsilon, \mathbf{X}_{s}^{\varepsilon}, \mathbf{Y}_{s}^{\varepsilon}\right) \mathrm{d} s
$$

where $\widehat{\mathrm{M}}^{\varepsilon}$ is a $\widehat{\mathbb{P}}^{\varepsilon}$-martingale, whose cross-variations are again given by (12). With the ergodic theorem, the cross-variations of $\widehat{\mathrm{M}}^{\varepsilon}$ converges in probability to $\bar{a}$ under $\mathbb{P}^{\varepsilon}$, hence under $\widehat{\mathbb{P}}^{\varepsilon}$. The Central Limit Theorem for martingale implies the existence of some $\widehat{\mathbb{P}}$-Brownian Motion $B$ such that $\widehat{M}^{\varepsilon}$ converges in distribution to $\bar{\sigma} \mathrm{B}$.

The functions $\nabla u_{i}$ and $x \mapsto \widetilde{h}(x / \varepsilon, \cdot, \cdot)$ are 1-periodic, and $\left(\mathcal{L}\left(\mathrm{X}^{\varepsilon}, \mathrm{Y}^{\varepsilon} \mid \widehat{\mathbb{P}}^{\varepsilon}\right)\right)_{\varepsilon>0}$ is known to converge. In fact, there exists some deterministic functions $(x, y)$ such that $D_{t}^{i}=\int_{0}^{t} \nabla u_{i}\left(\mathbf{X}^{\varepsilon} / \varepsilon\right) \cdot \widehat{h}\left(s, \mathbf{X}_{s}^{\varepsilon} / \varepsilon, \mathbf{X}^{\varepsilon}, \mathbf{Y}_{s}^{\varepsilon}\right) \mathrm{d} s$ is "close" to $\int_{0}^{t} \nabla u_{i}\left(\mathbf{X}^{\varepsilon} / \varepsilon\right)$. $\widehat{h}\left(s, \mathrm{X}_{s}^{\varepsilon} / \varepsilon, x_{s}, y_{s}\right) \mathrm{d} s$. With the help of the ergodic theorem, the term $D_{t}^{i}$ converges in probability under $\widehat{\mathbb{P}}^{\varepsilon}$ to $\int_{0}^{t} \int_{\mathbb{T}^{N}} \nabla w_{i}(x) \cdot \widehat{h}\left(s, x, \overline{\mathrm{X}}_{s}, \overline{\mathrm{Y}}_{s}\right) \mathrm{d} x \mathrm{~d} s=$ $\int_{0}^{t} \overline{\widehat{h}}_{i}\left(s, \overline{\mathrm{X}}_{s}, \overline{\mathrm{Y}}_{s}\right) \mathrm{d} s$ 
Then, the process $\mathcal{L}\left(\mathrm{X}^{\varepsilon} \mid \widehat{\mathbb{P}}^{\varepsilon}\right)$ converges to the distribution of the solution of the SDE

$$
\overline{\mathrm{X}}_{t}=x+\bar{\sigma} \mathrm{B}_{t}+\int_{0}^{t} \overline{\hat{h}}\left(s, \overline{\mathrm{X}}_{s}, \overline{\mathrm{Y}}_{s}\right) \mathrm{d} s .
$$

Step 4: Passage to limit of the BSDE. In fact, it may also be proved that $\bar{Y}$ satisfies

$$
\overline{\mathrm{Y}}_{t}=g\left(\overline{\mathrm{X}}_{T}\right)+\int_{t}^{T} \bar{h}\left(s, \overline{\mathrm{X}}_{s}, \overline{\mathrm{Y}}_{s}\right) \mathrm{d} s+\overline{\mathrm{M}}_{T}-\overline{\mathrm{M}}_{t}
$$

where

$$
\bar{h}(t, x, y)=\int_{\mathbb{T}^{N}} h(t, z, x, y) \mathrm{d} z
$$

Step 5: Identification of the limit. The limit martingale $\bar{M}$ and the Brownian Motion $B$ are both martingales with respect to the filtration generated by $\bar{X}, \bar{Y}$ and $\bar{M}$. Hence, if $(Y, Z)$ is the unique solution of

$$
\mathrm{Y}_{T}=g\left(\overline{\mathbf{X}}_{T}\right)+\int_{t}^{T} \bar{h}\left(s, \overline{\mathbf{X}}_{s}, \mathbf{Y}_{s}\right) \mathrm{d} s-\int_{t}^{T} \bar{\sigma}^{\mathrm{T}} \mathbf{Z}_{s} \mathrm{~dB}
$$

the the Itô formula applied to $|\mathrm{Y}-\overline{\mathrm{Y}}|^{2}$ and the Gronwall Lemma implies that $\overline{\mathrm{Y}}=\mathrm{Y}$ and $\overline{\mathrm{M}}=\bar{\sigma} \mathrm{B}$.

Step 6: Final identification. Again by some Girsanov transform, There exists a distribution $\mathbb{P}$ under which $\left(\mathrm{B}_{t}-\bar{\sigma}^{-1} \int_{0}^{t} \bar{h}\left(s, \overline{\mathrm{X}}_{s}, \mathrm{Y}_{s}\right) \mathrm{d} s ; t \geqslant 0\right)$ is a Brownian Motion. Under this probability $\mathbb{P}$, the processes $\left(\mathrm{Y}_{t}, \mathrm{Z}_{t}\right)_{t \in[0, T]}$ are solutions to the BSDE

$\mathbf{Y}_{t}=g\left(\overline{\mathbf{X}}_{T}\right)+\int_{t}^{T} \bar{h}\left(s, \overline{\mathbf{X}}_{s}, \mathbf{Y}_{s}\right) \mathrm{d} s+\int_{t}^{T} \mathbf{Z}_{s} \bar{h}\left(s, \overline{\mathbf{X}}_{s}, \mathbf{Y}_{s}\right) \mathrm{d} s-\int_{t}^{T} \bar{\sigma}^{\mathrm{T}} \mathbf{Z}_{s} \mathrm{~dB}{ }_{s}$.

Step 7: The final step. In may be proved that in fact $Y_{0}^{\varepsilon}$ converges in to $Y_{0}$, which is not clear at first sight because we have proved only the convergence of $Y^{\varepsilon}$ to $Y$ in the $S$-topology, which does not implies the convergence of $Y_{t}^{\varepsilon}$ to $Y_{t}$ for a fixed $t$. But in this case, this is true for $t=0$. Hence, since $Y_{0}^{\varepsilon}$ gives a version to the solution $u^{\varepsilon}$ of the semi-linear PDE (5), we have then proved that $u^{\varepsilon}$ converges pointwise to the solution of the semi-linear PDE (9).

Remark. The previous results may easily be extended to the case of systems, or for elliptic semi-linear PDEs. 
A. Lejay / Weak solution of semi-linear PDE, BSDE and homogenization

\section{References}

[BH98] P. BRIAND and Y. HU. Stability of BSDEs with random terminal time and homogeneization of semilinear elliptic PDEs. J. Funct. Anal., 155:455-494, 1998. 3

[BHP98] R. BUCKDAHN, Y. HU, and S. PENG. Probabilistic approach to homogenization of viscosity solutions of parabolic PDEs. NoDEA Nonlinear Differential Equations Appl., 6:395-411, 1999. 3

[BL97] G. BARLES and E. LESIGNE. SDE, BSDE and PDE, pages 47-80. Pitman Research Notes in Mathematics Series. Longman, 1997. 4

[BLP78] A. BENSOUSSAN, J.L. LIONS, and G. PAPANICOLAOU. Asymptotic Analysis for Periodic Structures. North-Holland, 1978. 3

[BPS99] V. BALLY, É. PARDOUX, and L. STOICA. Backward stochastic differential equations associated to a symmetric Markov process. Potential Anal., 22(1):17-60, 2005. 4

[BRI97] P. BRIAND. Équations différentielles stochastiques rétrogrades: applications aux équations aux dérivées partielles. $\mathrm{PhD}$ thesis, Université Blaise-Pascal, 1997. 3

[CAS00] F. CASTELL. Homogenization of random semilinear PDEs. Probab. Theory Related Fields, 121(4):492-524, 2001. 3

[EK86] S.N. ETHIER and T.G. KURTZ. Markov Processes, Characterization and Convergence. Wiley, 1986. 6

[EK97] N. EL KAROUI. Backward stochastic differential equations: a general introduction, pages 7-26. Pitman Research Notes in Mathematics Series. Longman, 1997. 2

[FOT94] M. FUKUSHIMA, Y. OSHIMA, and M. TAKEDA. Dirichlet Forms and Symmetric Markov Process. De Gruyter, 1994. 4, 5

[GAU99] G. GAUDRON. Convergence en loi d'EDS et d'EDS Rétrogrades. Application à l'homogénéisation d'EDP linéaires ou semilinéaires. PhD thesis, Université de Provence, 1999. 3 
A. Lejay / Weak solution of semi-linear PDE, BSDE and homogenization

[HP97] Y. HU and S. PENG. A stability theorem of backward stochastic differential equations and its application. Comptes-rendus à l'Académie des Sciences, 324:1059-1064, 1997. Série 1. 3

[HU97] Y. HU. Stability theorems and homogenization of nonlinear PDEs with periodic structures, pages 193-205. Pitman Research Notes in Mathematics Series. Longman, 1997. 3

[JAK97] A. JAKUBOWSKI. A non-skorohod topology on the skorohod space. Electronic Journal of Probability, 2, 1997. http://www. math. washington. edu/ ejpecp/. 3,6

[LEJ00a] A. LEJAY. BSDE driven by Dirichlet process and semi-linear parabolic PDE. application to homogenization. Stochastic Process. Appl., 97(1):1-39, 2002. 4

[LEJ00b] A. LEJAY. Méthodes probabilistes pour l'homogénéisation des opérateurs sous forme-divergence : cas linéaires et semi-linéaires. PhD thesis, Université de Provence, 2000. 4

[LSU68] O.A. LADYŽENSKAJA, V.A. SOLONNIKOV, and N.N. URAL'CEVA. Linear and Quasilinear Equations of Parabolic Type. American Mathematical Society, 1968. 4

[MR91] Z. MA and M. RÖCKNER. Introduction to the Theory of (NonSymmetric) Dirichlet Forms. Universitext. Springer-Verlag, 1991. 4

[PAR98] E. PARDOUX. Stochastic analysis and related topics: The Geilo workshop 1996. In L. Decreusefond, J. Gjerd, B. Øksendal, and A.S. Ustünel, editors, Stochastic Analysis and Related Topics: The Geilo Workshop, 1996, pages 79-127. Birkhäuser, 1998. 4

[PAR99a] É. PARDOUX. BSDEs, weak convergence and homogenization of semilinear PDEs. In F.H. Clarke and R.J. Stern, editors, Non Linear Analysis, Differential Equations and Control. Proc. Séminaire de Mathématiques Supérieures, Montréal 1998, pages 503549. Kluwer, 1999. 2, 6

[PAR99b] É. PARDOUX. Homogenization of linear and semilinear second order PDEs with periodic coefficients: a probabilistic approach. J. Funct. Anal., 167(2):498-520, 1999. 3 
A. Lejay / Weak solution of semi-linear PDE, BSDE and homogenization

[PO99] É. PARDOUX and Y. OUKNINE. Homogenization of PDE's with non linear boundary condition. In Proc. Conf. Ascona, 1999, 1999. To appear. 3

[PP90] É. PARDOUX and S. PENG. Adapted solution of a backward stochastic differential equation. Systems $\&$ Control Letters, 14:5561, 1990. 2

[PV97] É. PARDOUX and A.Y. VERETENNIKOV. Averaging of backward SDEs, with application to semi-linear PDEs. Stochastics and Stochastics Reports, 60:255-270, 1997. 3,6 